\title{
An Optimal Distribution of RSU for Improving Self-Driving Vehicle Connectivity
}

\author{
Khattab Alheeti ${ }^{1}$, Abdulkareem Alaloosy ${ }^{1}$, Haitham Khalaf ${ }^{2}$, Abdulkareem Alzahrani ${ }^{3, *}$ \\ and Duaa Al_Dosary ${ }^{4}$
}

\author{
${ }^{1}$ Department of Computer Networking System, College of Computer Sciences and Information Technology, University of \\ Anbar, Ramadi, Iraq \\ ${ }^{2}$ College of Medicine, University of Anbar, Ramadi, Iraq \\ ${ }^{3}$ Department of Computer Science, Faculty of Computer Science and Information Technology, Albaha University, Albaha, \\ Saudi Arabia \\ ${ }^{4}$ College of Computer Sciences and Information Technology, University of Anbar, Ramadi, Iraq \\ ${ }^{*}$ Corresponding Author: Abdulkareem Alzahrani. Email: ao.alzahrani@bu.edu.sa \\ Received: 25 April 2021; Accepted: 04 July 2021
}

\begin{abstract}
Self-driving and semi-self-driving cars play an important role in our daily lives. The effectiveness of these cars is based heavily on the use of their surrounding areas to collect sensitive and vital information. However, external infrastructures also play significant roles in the transmission and reception of control data, cooperative awareness messages, and caution notifications. In this case, roadside units are considered one of the most important communication peripherals. Random distribution of these infrastructures will overburden the spread of self-driving vehicles in terms of cost, bandwidth, connectivity, and radio coverage area. In this paper, a new distributed roadside unit is proposed to enhance the performance and connectivity of these cars. Therefore, this approach is based primarily on $k$-means to find the optimal location of each roadside unit. In addition, this approach supports dynamic mobility with a long period of connectivity for each car. Further, this system can adapt to various locations (e.g., highways, rural areas, urban environments). The simulation results of the proposed system are reflected in its efficiency and effectively. Thus, the system can achieve a high connectivity rate with a low error rate while reducing costs.
\end{abstract}

Keywords: Self-driving cars; roadside unit; autonomous vehicles; distributed systems; connectivity

\section{Introduction}

Vehicular ad hoc networks or VANETs are a technology that provides wireless communications of self-driving vehicles. VANETs represent a group of wireless mobile vehicles as router or node allowing them to communicate and create a network with vehicle radio range [1]. Dedicated Short-Range Communications (DSRC) is a new wireless technology utilized by VANETs when sending traffic information, safety messages, and road information then forwarded to vehicles [2]. In VANETs, vehicles communicate wirelessly with one another which called Vehicle-to-Vehicle

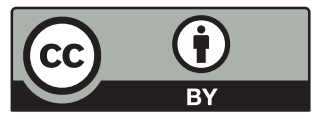

This work is licensed under a Creative Commons Attribution 4.0 International License, which permits unrestricted use, distribution, and reproduction in any medium, provided the original work is properly cited. 
(V2V) communication. Besides the V2V type of VANET, wireless communication can be created between the vehicles and their fixed infrastructure. This communication called Vehicle-toInfrastructure (V2I) that is useful in management services and traffic settings [3]. Fig. 1 presents a communication system for self-driving vehicles.

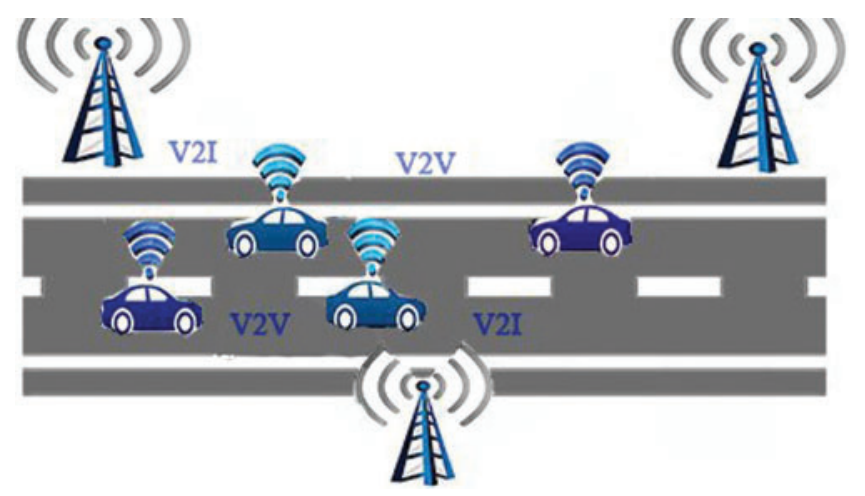

Figure 1: Communication system of autonomous vehicles

Intelligent transportation systems added the capabilities of wireless communication to VANET for safety and to protect drivers, passengers, and vehicles [1]. To ensure these wireless communication capabilities stay constant, efficient roadside infrastructures for communication are required. These roadside units (RSU) are generally managed and funded by national governments and come at a high cost. In addition, the number of accidents increases as the number of vehicles increases [4]. Providing safety protocols for transportation systems is essential; thus, VANET play a vital role because they eliminate space and time requirements for vehicles and make wireless information communication available to the vehicles when necessary.

Keeping passengers and drivers safe is the main goal of self-driving vehicles. These vehicles reduce traffic accidents resulting from human error. Self-driving vehicles rely on communication systems to exchange sensitive information with RSU. The latter are nodes installed within infrastructure that are used for wireless communication. A vehicle's capacity for communication with an infrastructure depends on the number of RSU. However, the process of installing and maintaining RSU costs considerable money; therefore, authorities aim to decrease the number of RSU, thus making such units an invaluable resource in VANET.

In this paper, we propose a new distributed RSU to develop car performance and connectivity. The proposed system aims to find the optimal location of each RSU by utilizing the $k$-means algorithm. This system can present numerous advantages in terms of better connectivity rate and reduced cost.

The remaining sections in the paper are: related works, $k$-means algorithms, methodology of the proposed system, simulation results, and conclusions and future directions.

\section{Related Works}

Several studies have been presented to determine the number of RSU required to provide sufficient deployment for connectivity within a given scenario [5]. The authors proposed a cumulative weight-based system that aims to provide effective distribution of RSU to improve the efficiency and quality of transportation systems. The process of installing and maintaining RSU 
requires considerable funding; therefore, authorities want to decrease the number of RSU. In [6], the authors proposed a system to achieve less cost to connect with emergency services when an accident occurs; their work is based on distributing RSU by depending on an inverse proportion to the density of the predicted vehicles. Simulation results prove when the number of RSU is decreased, so is the time required to notify emergency services of an accident. In [7], the authors aimed to improve the overall network performance and reduce the number of RSU; they also proposed a new algorithm for the deployment of RSU. Simulation results proved the proposed algorithm can satisfy the lowest RSU deployment cost.

In [8], the authors aimed to improve wireless communication between the vehicles and RSU when sending data to reduce latency time; their simulation results proved packet loss and latency time are reduced, and the range of the network has been increased tremendously by using WiMax technology. In [2], the authors proved connectivity and message reachability were increased by a system that used a self-organized network when specific vehicles represented RSU. The system depended on proposed local rules and algorithms that adhere to such rules. To reduce the disconnection interval for RSU and improve transition rate and connectivity, [9] proposed a placement scheme capable of organizing road networks based on a vehicle's real-time movement, RSU, and transmission range. Simulation results proved while the disconnection time was preserved below $10 \mathrm{~s}$ and $1,000 \mathrm{RSU}, 72.5 \%$ of connectivity can be assured when the transition rate is $300 \mathrm{~m}$. The authors in [10] proposed a system based on using a genetic algorithm to provide an automatic $\mathrm{RSU}$; their simulation results showed the proposed system was capable of decreasing the notification time of an accident and vehicular communication within various density scenarios. [11] further proposed a genetic algorithm for optimizing the RSU's positions. Simulation results of the genetic algorithm proved the viability of this system by introducing achievable improvements to the VANET model. In [12], the authors accounted for the number of vehicles and their speed for locating roadside infrastructure by depending on the evolutionary algorithm; they aimed to decrease the RSU deployment cost. Simulation results proved the proposed algorithm was capable of determining precise trade-off solutions for the problem. In [13], the authors used Time Threshold Problem (MCTTP) for the deployment of RSU with a genetic algorithm. Simulation results proved the proposed system offered good results and presented up to 11 percentage points.

Our proposed system differs from earlier works in that it uses the $k$-means algorithm to find a new distributed RSU to enhance cars' performance and connectivity. Our system aims to present a high connectivity rate and to reduce costs.

\section{K-Means Algorithm}

In a vehicular network, $k$-means is an unsupervised knowledge learning and clustering method used to find the solution to numerous problems. $k$-means is a powerful algorithm because of its scalability, simplicity, and capability of adapting to different request domains and scenarios.

$k$-means is a partitioning algorithm that collects nodes to generate the $k$ of groups by dividing $n$ of data. It identifies $k$ number of centroids and then allocates each data point to the nearest cluster while keeping the centroids as small as possible. The $k$-means algorithm aims to select $k$ centroids where each centroid belongs to one cluster. The initial centroid is selected randomly and then used to determine the next cluster of centroids, as shown in Eq. (1) [14]:

$J=\sum_{n=1}^{N} \sum_{k=1}^{K} r_{n k}\left\|X_{n}-\mu_{k}\right\|^{2}$ 
By applying Eq. (1), the distance between the node and the center of the cluster, represented by $J$, is calculated; $K$ is the number of clusters, $N$ is the number of nodes, and $\mu_{\mathrm{k}}$ is the position of $k$-means.

There are five iterative steps that characterizes the way $k$-mean algorithm works; these steps are:

(a) Identify the desired number of clusters $k$.

(b) Select the centroid for each cluster.

(c) Assign each data point to the closest cluster centroid.

(d) Compute the centroids of newly formed clusters.

(e) Repeat steps c and d.

\section{Methodology of the Proposed System}

In this paper, we propose a new system to distribute the RSU. The proposed system is based heavily on the $k$-means algorithm to find the optimal location for each RSU. The system aims to enhance performance and connectivity and reduce costs while decreasing the number of RSU. The methodology is explained in the sections below.

\subsection{System Scenario}

A new distributed RSU is proposed to enhance connectivity and performance in a VANET environment. The proposed system is based significantly on $k$-means to find the optimal location of each RSU. In addition, it will support dynamic mobility with a long period of connectivity for each car. The proposed system has the ability to adapt to an urban environment. Fig. 2 depicts the steps of our proposed system. These steps can be summarized as follows:

(a) Send data from RSU to the sensors in self-driving vehicles.

(b) Establish a simulation environment of VANET by ns-2.

(c) Determine the number of RSU by default $=100 \mathrm{RSU}$.

(d) Establish the coordination or dimension of the city by $x, y$.

(e) Use $k$-means to distribute RSU.

(f) Implement a training phase to get an optimal number of RSU.

(g) Gather results of the optimal number of RSU.

\subsection{Simulation Environment}

In this system, ns-2 is used to establish the VANET environment through the network animator (NAM) file generated as the output of the ns-2 simulation, as shown in Fig. 3.

In the begging, the dimensions of the city was identified as $x=5 \mathrm{~km}$ and $y=5 \mathrm{~km}$. The system consists of $100 \mathrm{RSU}$ on an ns-2 simulator. In the simulation system, initial parameters are an important factor; they specify the behavior and performance of ns-2. Tab. 1 shows the parameters used in simulating the VANET environment. 


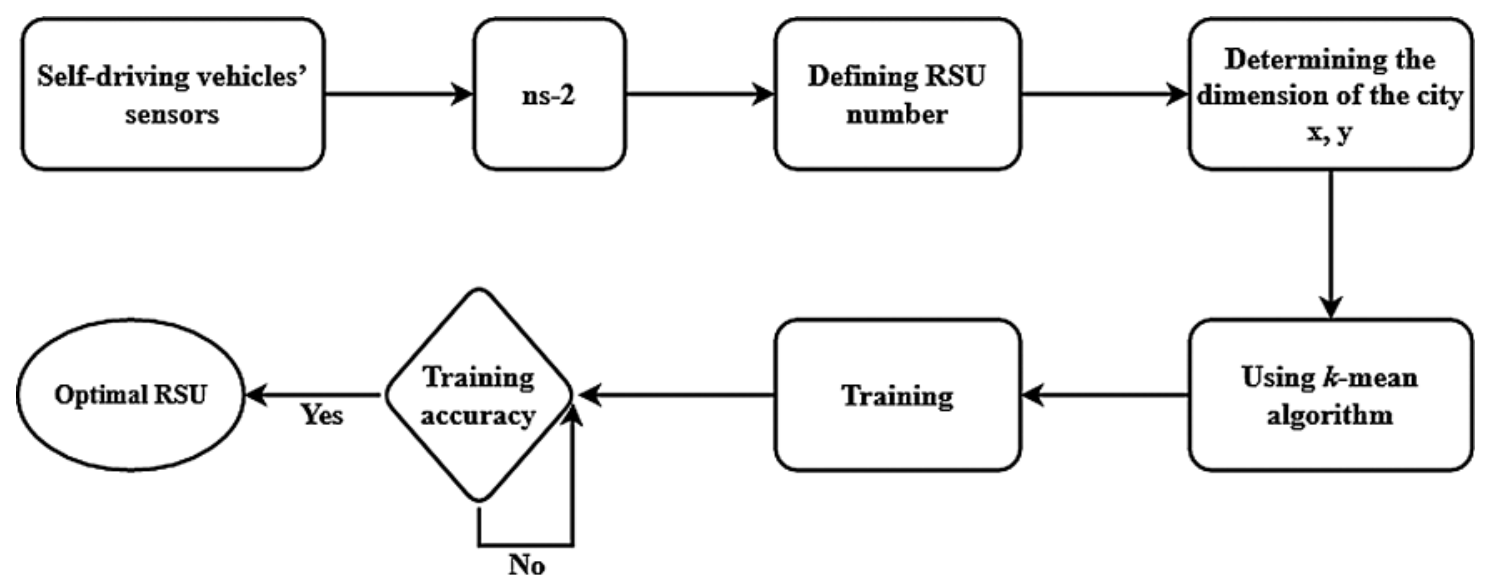

Figure 2: Architecture of the proposed system

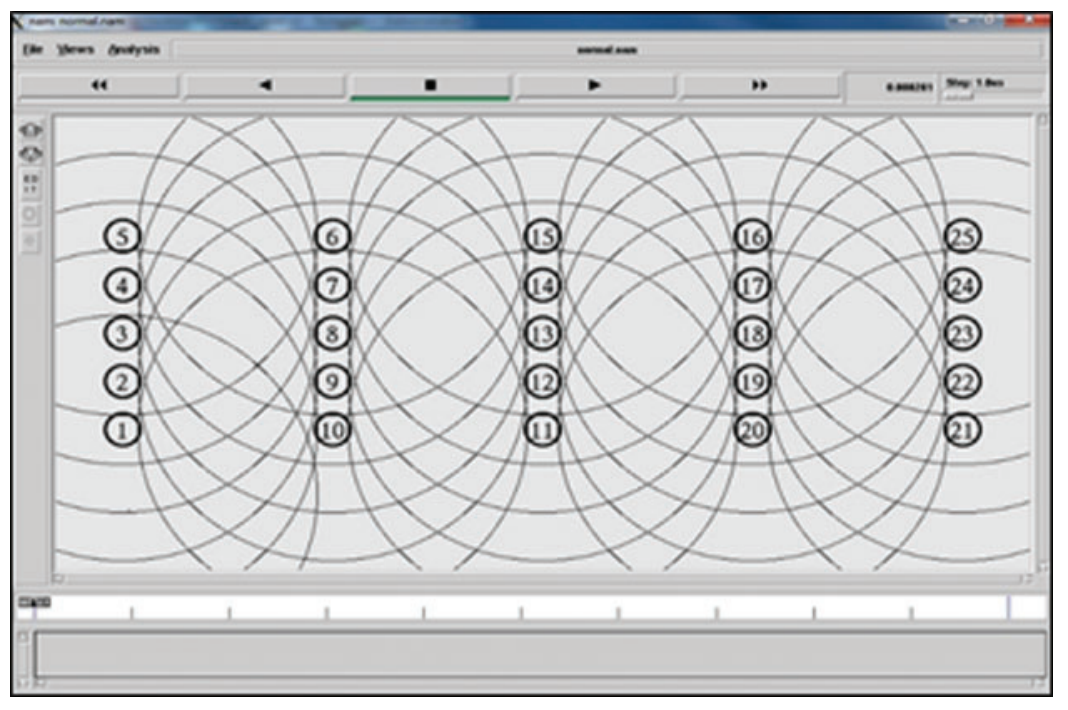

Figure 3: Simulation of NAM file in ns-2

Table 1: The parameters of simulation in ns-2

\begin{tabular}{ll}
\hline Parameter & Value \\
\hline RSUs number & 100 RSUs \\
Mobility models & Manhattan mobility model \\
Channel type & Wireless \\
Simulation time & $250 \mathrm{~s}$ \\
Traffic type & Constant bit rate $(\mathrm{CBR})$ \\
Topology & $5 \times 5(\mathrm{~km})$ \\
MAC protocol & IEEE 802.11 \\
Transport protocol & UDP \\
\hline
\end{tabular}




\section{Simulation Results}

In this section, we present the results obtained by this proposal. First, Tab. 2 presents the number of RSU with various dimensions. The results prove the ability of the proposed communication system to reduce the number of RSU when compared with traditional systems in different radio coverage area dimensions. Simulation results prove the proposed system has a reduction rate of $22.4 \%$ for the number of RSU.

Table 2: The parameters of simulation in ns-2

\begin{tabular}{lll}
\hline $\begin{array}{l}\text { Radio coverage } \\
\text { area dimensions }\end{array}$ & $\begin{array}{l}\text { Number of RSU } \\
\text { with a traditional } \\
\text { system }\end{array}$ & $\begin{array}{l}\text { Number of RSU } \\
\text { with our proposal }\end{array}$ \\
\hline $5 \times 5(\mathrm{~km})$ & 25 & 20 \\
$10 \times 10(\mathrm{~km})$ & 100 & 83 \\
$15 \times 15(\mathrm{~km})$ & 225 & 167 \\
$20 \times 20(\mathrm{~km})$ & 400 & 311 \\
$25 \times 25(\mathrm{~km})$ & 625 & 499 \\
$30 \times 30(\mathrm{~km})$ & 900 & 686 \\
Total of RSU & 2275 & 1766 \\
\hline
\end{tabular}

Our proposed system has the ability to distribute $20 \mathrm{RSU}$ in $5 \times 5(\mathrm{~km})$ dimensions instead of $25 \mathrm{RSU}$, as shown in Tab. 2. Fig. 4 shows the position of RSU with a traditional system.

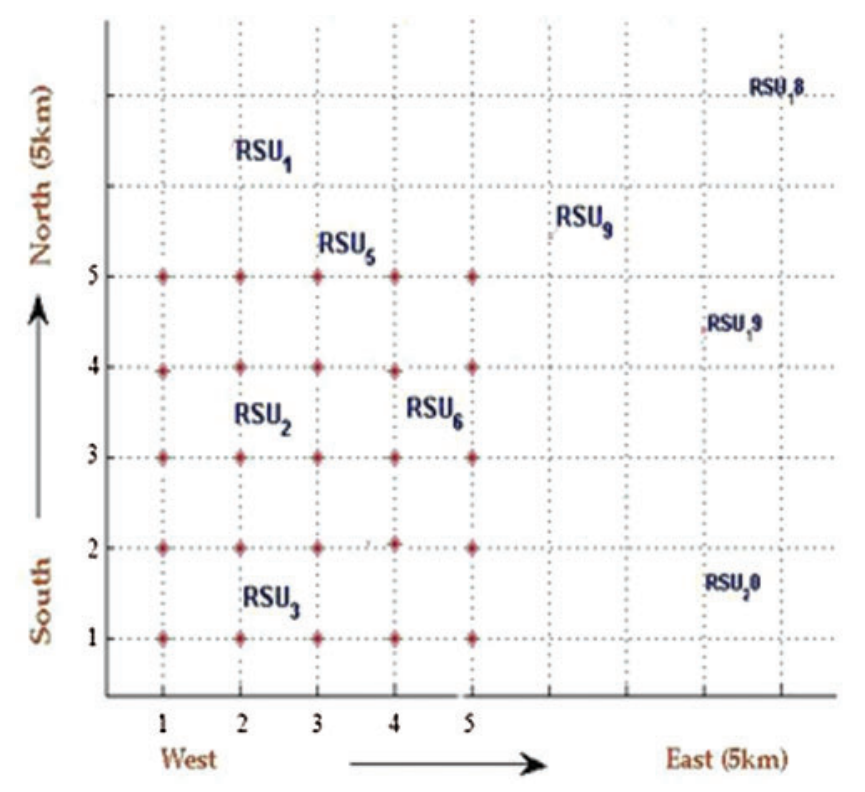

Figure 4: Position of RSU with a traditional system 
Fig. 5 shows the positions of RSU under the proposed system. The figure proves our proposal has the ability to distribute RSU in a way that can enhance performance and connectivity as well as reduce costs.

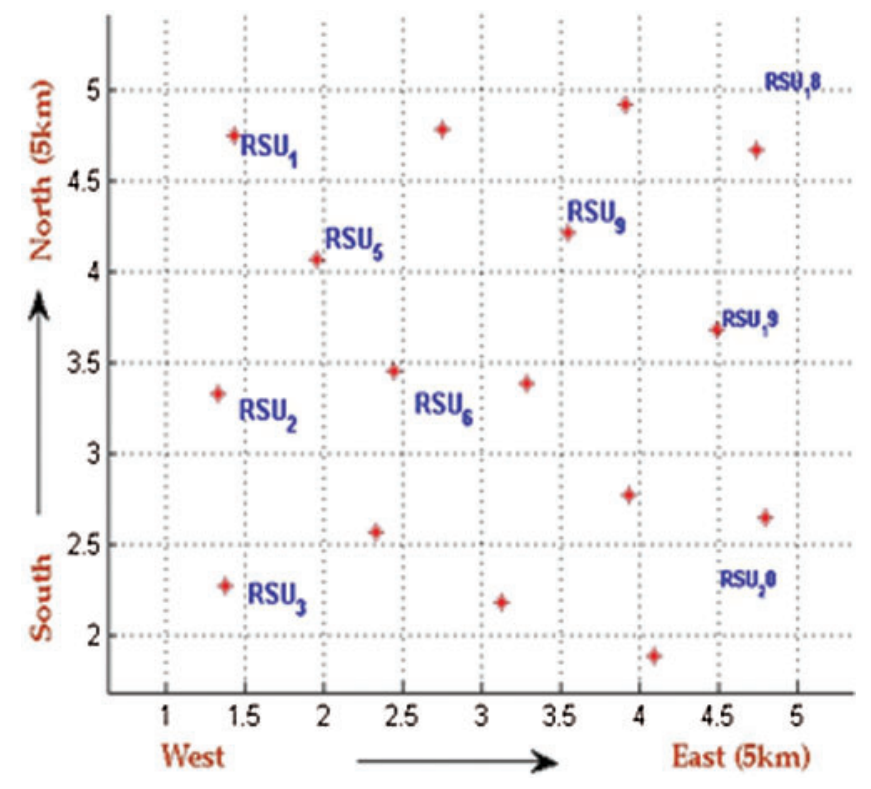

Figure 5: New position of RSU with the proposed system

Other performance metrics for system evaluation are studied and compared, including packet delivery ratio (PDR), throughput rate, and end-to-end delay average [3]. These metrics can be calculated by the following Eqs. (2)-(4):

$P D R=\sum N_{r} / \sum N_{S}$

where, $N_{r}=$ number of the packet received and $N_{s}=$ number of the packet sent.

Throughput $(\mathrm{kbps})=N_{r} * S / S T$

where, $N_{r}=$ number of the packet received and $S=$ packet size and $S T=$ simulation time.

End-to-End Delay $(\mathrm{ms})=\left(\frac{\sum \text { end }_{\text {time }}-\text { start }_{\text {time }}}{\sum N}\right)$

where $N$ represent the number of connections.

Tab. 3 presents the performance metrics of the proposed communication system and compares these metrics with the traditional communication system.

Although Tab. 2 shows the proposed system has good results in reducing the number of RSU in different dimensions, the proposed system has reasonable results regarding other communication metrics, as presented in Tab. 3. 
Table 3: The performance metrics

\begin{tabular}{lll}
\hline $\begin{array}{l}\text { Performance } \\
\text { evaluation criteria }\end{array}$ & $\begin{array}{l}\text { Self-driving vehicles } \\
\text { with traditional } \\
\text { communication } \\
\text { system }\end{array}$ & $\begin{array}{l}\text { Self-driving vehicles } \\
\text { with proposed } \\
\text { communication } \\
\text { system }\end{array}$ \\
\hline Packet delivery rate $(\%)$ & 87.32 & 84.6 \\
End-to-End delay $(\mathrm{ms})$ & 1.23 & 1.7 \\
Throughput rate $(\mathrm{kpbs})$ & 76.4 & 74.8 \\
\hline
\end{tabular}

\section{Conclusions and Future Directions}

A new class of vehicles known as self-driving and semi-self-driving cars plays an important role in VANET. These cars cooperate, send, and receive data packets to one another wirelessly through the cars' radio range. External infrastructures that represent RSU are needed for communication. Random distribution of RSU will be costly and will overburden bandwidth and connectivity over the radio coverage area. In this paper, we propose a new distribution of RSU to enhance these cars' performance and connectivity. This approach is based heavily on $k$-means to find the optimal location of each RSU. The proposed system can reduce costs and achieve a high connectivity rate with a reduction rate of $22.4 \%$ for RSU numbers. A possible future function of the system is dealing with various artificial intelligence techniques (e.g., a genetic algorithm) to find and distribute the optimal number of RSU.

Funding Statement: The authors received no specific funding for this study.

Conflicts of Interest: The authors declare that they have no conflicts of interest to report regarding the present study.

\section{References}

[1] K. M. Ali Alheeti, A. Gruebler and K. D. McDonald-Maier, "An intrusion detection system against malicious attacks on the communication network of driverless cars," in 2015 12th Annual IEEE Consumer Communications and Networking Conf., Las Vegas, NV, USA, IEEE, pp. 916-921, 2015.

[2] O. K. Tonguz and W. Viriyasitavat, "Cars as roadside units: A self-organizing network solution," IEEE Communications Magazine, vol. 51, no. 12, pp. 112-120, 2013.

[3] K. M. Ali Alheeti and K. McDonald-Maier, "An intelligent intrusion detection scheme for selfdriving vehicles based on magnetometer sensors," in 2016 Int. Conf. for Students on Applied Engineering, Newcastle Upon Tyne, UK, IEEE, pp. 75-78, 2016.

[4] F. J. Martinez, C. Toh, J. Cano, C. T. Calafate and P. Manzoni, "Emergency services in future intelligent transportation systems based on vehicular communication networks," IEEE Intelligent Transportation Systems Magazine, vol. 2, no. 2, pp. 6-20, 2010.

[5] A. Makkawi, R. Daher and R. Rizk, "RSUs placement using cumulative weight based method for urban and rural roads," in 2015 7th Int. Workshop on Reliable Networks Design and Modeling, Munich, Germany, IEEE, pp. 307-313, 2015.

[6] J. Barrachina, P. Garrido, M. Fogue, F. J. Martinez, J. Cano et al., "Road side unit deployment: A density-based approach," IEEE Intelligent Transportation Systems Magazine, vol. 5, no. 3, pp. 30-39, 2013. 
[7] Y. Liu, J. Ma, J. Niu, Y. Zhang and W. Wang, "Roadside units deployment for content downloading in vehicular networks," in 2013 IEEE Int. Conf. on Communications, Budapest, Hungary, IEEE, pp. 63656370, 2013.

[8] P. Shrivastava, S. Ashai, A. Jaroli and S. Gohil, "Vehicle-to-road-side-unit communication using Wimax," International Journal of Engineering Research and Applications, vol. 2, no. 4, pp. 1653-1655, 2012.

[9] J. Lee and C. M. Kim, "A roadside unit placement scheme for vehicular telematics networks," in Advances in Computer Science and Information Technology. AST 2010, ACN 2010. Lecture Notes in Computer Science. Berlin, Heidelberg: Springer, pp. 196-202, 2010.

[10] M. Fogue, J. A. Sanguesa, F. J. Martinez and J. M. Marquez-Barja, "Improving roadside unit deployment in vehicular networks by exploiting genetic algorithms," Applied Sciences, vol. 8, no. 1, pp. 86, 2018.

[11] C. Lochert, B. Scheuermann, C. Wewetzer, A. Luebke and M. Mauve, "Data aggregation and roadside unit placement for a vanet traffic information system," in Proc. of the Fifth ACM Int. Workshop on Vehicular Inter-Networking, New York City, NY, USA, Association for Computing Machinery (ACM), pp. 58-65, 2008.

[12] R. Massobrio, S. Bertinat, S. Nesmachnow, J. Toutouh and E. Alba, "Smart placement of RSU for vehicular networks using multiobjective evolutionary algorithms," in 2015 Latin America Congress on Computational Intelligence, Curitiba, Brazil, IEEE, pp. 1-6, 2015.

[13] E. S. Cavalcante, A. L. L. Aquino, G. L. Pappa and A. A. F. Loureiro, "Roadside unit deployment for information dissemination in a VANET: An evolutionary approach," in Proc. of the 14th Annual Conf. Companion on Genetic and Evolutionary Computation, Philadelphia, Pennsylvania, USA, Association for Computing Machinery (ACM), pp. 27-34, 2012.

[14] C. M. Bishop, Pattern Recognition and Machine Learning (Information Science and Statistics). Berlin, Heidelberg: Springer, 2006. 